\title{
Design of genetic sensors to ethylene based on bioinformatics analysis of whole-genome data
}

\author{
V. Dolgikh \\ Novosibirsk State University, Novosibirsk, Russia \\ e-mail:volleydollmc1@gmail.com
}

Key words: ethylene, ETHYLENE-INSENSITIVE3 (EIN3), ChIP-seq, RNA-seq, Arabidopsis thaliana

Motivation and Aim: Ethylene is a gaseous hormone that regulates a wide range of physiological processes involved in plant growth, development and stress responses. Ethylene signaling proceeds via a linear pathway leading to the stabilization of EIN3 transcription factor - the major transcriptional regulator of ethylene response. It activates the expression of a large pool of genes upon binding to the specific sites in their promoters. However, in many cases EIN3 binding to gene promoter is not enough to trigger transcriptional response, which unveils the complexity of EIN3-dependent regulation [1]. Ethylene sensitive Arabidopsis thaliana reporter lines are important tools to localize the domains of ethylene biosynthesis in plants. The available genetic sensors used to date utilize several copies of EIN3 binding sites from direct EIN3 targets EDF1 or ERF1 inserted upstream of a minimal promoter followed by a reporter gene $[2,3]$. However, it is quite possible that the reporter expression pattern in these lines does not strictly match the domain of ethylene biosynthesis due to the mentioned above complexity of EIN3-promoted regulation. Here we perform the bioinformatics analysis of whole genome data on EIN3 binding coupled with the analysis of ethylene-induced transcriptomes in A. thalliana to design a set of genetic sensors to ethylene, which strictly define the domains ethylene biosynthesis.

Methods and Algorithms: We used publicly available ChIP-seq and RNA-seq data from [1] for bioinformatics analysis. Regulatory elements were identified de novo with Homer tool [4]. The significance of motif enrichment in ethylene responsive genes was determined with Fisher's exact test.

Results: We distinguished several EIN3 regulatory patterns and coupled them with ethylene-induced gene expression. We used these predicted regulatory patterns to design a set of fluorescent genetic sensors to ethylene based on ethylene-induced GFP protein expression. The following experimental verification of these biosensors will expand our understanding of the issues that affect hormonal regulation in plants.

Acknowledgements: Supported by Russian Ministry of Science and Education under 5-100 Excellence Programme.

\section{References}

1. Chang K.N. et al. (2013) Temporal transcriptional response to ethylene gas drives growth hormone crossregulation in Arabidopsis. eLife. 2:e00675.

2. Stepanova A.N. et al. (2007) Multilevel interactions between Ethylene and Auxin in Arabidopsis roots. Plant Cell. 19(7):2169-2185.

3. Solano R. (1998) Nuclear events in ethylene signaling: a transcriptional cascade mediated by ETHYLENE-INSENSITIVE3 and ETHYLENE-RESPONSE-FACTOR1. Genes and Development. 12(23):3703-3714.

4. Heinz S. et al. (2010) Simple combinations of lineage-determining transcription factors prime cisregulatory elements required for macrophage and B cell identities. Mol Cell. 38:576-589. 